\title{
Microbial infection and antibiotic patterns among intensive care unit patients in a tertiary hospital in Central Nepal
}

\section{RK Sanjana, ${ }^{1}$ PC Majhi ${ }^{2}$}

${ }^{1}$ Assistant Professor, Department of Microbiology, ${ }^{2}$ Prof. \& Head, Department of Anaesthesiology and Critical care,College of Medical Sciences, Bharatpur, Chitwan, Nepal

\section{ABSTRACT}

Aims

The present study is a fundamental effort to evaluate the bacteriological and antibiotic patterns in the Intensive Care Unit (ICU) setting in a tertiary care hospital. This study was carried out to guide the clinician in choosing the appropriate antibiotics and to prevent emergence of multidrug resistance bacteria.

\section{Materials and Methods}

Between March 2009 to Feb 2012, the samples from various ICUs that were submitted to the microbiology laboratory for culture and sensitivity were included in this study. All the organisms were identified morphologically and biochemically by standard laboratory procedure and antibiotic susceptibility pattern was determined by disc diffusion methods.

\section{Result}

Of 3,780 specimens, 2,312 (61.1\%) isolates were recovered. Single organisms were isolated from 1,746 (75.5\%) samples while the remaining $566(24.4 \%)$ had two or more organisms isolated. Pseudomonas aeruginosa was the most common isolate 819 (35.4\%), followed by Klebsiella pneumoniae 637 (27.5\%). Antibiotics sensitivity of Pseudomonas aeruginosa showed that almost all isolates were resistant to cephalexin (97.8\%) and ciprofloxacin (80.3\%). However $(95.8 \%)$ of the isolates were sensitive to tobramycin, $(92.0 \%)$ to meropenem \& $(74.80 \%)$ to amikacin.

\section{Conclusion}

It is concluded that Pseudomonas aeruginosa is the predominate pathogen isolated from ICUs of this Hospital. Most of the frequently isolated pathogens are resistant to cephalosporins and quinolone antibiotics as compared to aminoglycosides and carbapenem. Regular surveillance of antibiotic susceptibility pattern is very important for setting a guideline to the clinician in choosing an appropriate therapy of infected patients of ICUs.

\section{Key words: Antibiotic resistance, intensive care unit, microbial infection.}

Correspondence: Dr. RK Sanjana

E-mail: rajkumari_sanjana@yahoo.co.in 


\section{Introduction}

Advances in medical technology and expertise over the past decades have resulted in the ability to maintain vital organ functions in critically ill patients until such a time as their vital functions has been re-established. By necessity these patients have been clustered in units that specialise in technique of intensive care and therapy. In the Intensive Care Unit (ICU), the accumulation of a number of immunocompromised patients and their nursing and invasive procedures provide a favourable environment to the growth and transmission of nosocomial infections. ${ }^{1,2,3,4,5}$

ICU is one of potential sources of nosocomial infections even in countries where extensive infection control measures are routinely implemented. The international study of infections in ICU which was conducted in 2007, and involved with 1,265 ICUs, from 75 countries, demonstrated that patients who had longer ICU stays had higher rates of infection, especially infections due to resistant Staphylococci, Acinetobacter, Pseudomonas species and Candida species. Moreover, the ICU mortality of infected patients was more than twice that of non infected patients. ${ }^{6}$ Most ICU patients that acquired infections were associated with the use of invasive devices such as catheter, endotracheal tubes and mechanical ventilators. ${ }^{7}$

Globally, patients in the ICUs have encountered an increasing emergence and spread of antibiotic resistant pathogens. Although ICUs generally comprise $5 \%$ of all hospital bed, they account for $20 \%$ to $25 \%$ of all nosocomial infections. ${ }^{8}$ The increased risk of infections is associated with the severity of the patient contact with healthcare personal and length of stay in the ICU. ${ }^{9}$
The parameters by which the infections were judged in these patients have had to be altered to such an extent that the bacteriology of intensive care has become virtually a sub- speciality within routine microbiology. Moreover, this factor may vary from one geographical region to the other in almost all cases; eradication of causative agents requires initiation of antimicrobial therapy before obtained culture report. However, during the last few years the increase in antibiotic resistance has compromised the selection of empirical complicated treatment. ${ }^{10}$

The knowledge of likely prevalent strains along with their antimicrobial resistance pattern will help in better management of patients and framing the antibiotic policy.

This study was conducted with the aim of evaluating the bacteriological profile and tracing resistance rate among different infections in the ICU patients in this hospital.

\section{Materials and methods}

The present retrospective study was carried out based on reports of bacterial isolates of various clinical specimens from different ICUs of College of Medical Sciences-Tteaching hospital, Bharatpur (COMS-TH) that were submitted to the Microbiology laboratory during the period of March 2009 to February 2012. A total number of 3,780 specimens were received during this period. Various sources of clinical specimens include blood, urine, pus, CSF, catheter tips, endotracheal tips, drainage fluids (trauma, pleural, ascitic) bronchial aspirates, central venous catheters (CVC) etc. 
RK Sanjana et al. Microbial infection and antibiotic patterns among

All the specimens received were immediately plated on the blood agar and Mac-Conkey agar and incubated aerobically overnight at $37^{\circ} \mathrm{C}$. Single and mixed growth (two or more than two isolates) per specimens isolated from all the sample were identified by observing the colony characteristics on blood agar, Mac-Conkey agar plates and biochemical reactions using standard microbiological method. ${ }^{11}$

Antibiotic susceptibility testing was done by disc diffusion method..$^{12}$ The following antibiotics (Hi-media disc in $\mu \mathrm{g}$ ) were tested : ampicillin (10), cephalexin (30), cefotaxime (30), amoxycillin/clavulanic acid (20/ 10), piperacillin (100), ceftazidime (30), ceftriaxone (30), meropenem (10), gentamicin(10), amikacin (30), ciprofloxcian (5), nitrofurantoin (100), cotrimoxazole (25), levofloxacin (5), cefepime(30). Zone of diameter was measured and interpreted as per the Clinical and Laboratory Standard Institute (CLSI) guidelines. ${ }^{12}$ For quality control of disc diffusing tests, ATCC control strains of Staphylococcus ATCC 43300, Escherichia coli ATCC 25922 and Pseudomonas aeruginosa ATCC 27873 strains were used.

\section{Results}

A total of 3,780 samples were received during the period of March 2009 to Feb 2012.

Out of the total samples received, organisms were isolated from 2,312 (61\%).One thousand seven hundred and forty six samples (75.5\%) had single organism, while the remaining five hundred and sixtysix (24.4\%) had two or more organisms isolated. There were one thousand four hundred and sixty eight (38.8\%) samples that had a sterile culture with no organism isolated. The most frequently isolated bacteria were Pseudomonas aeruginosa (P. aeruginosa) 819 (35.4\%) and Klebsiella pneumoniae (K. pneumonia) 637(27.5\%), followed by Acinetobacter anitratus (A. anitratus) 368 (15.9\%), Enterobacter species 144(6.2\%), Escherichia coli (E. coli) 128 (5.5\%), Proteus mirabilis (P. mirabilis) 81(3.5\%), Staphylococcus aureus (S. aureus) 53(2.2\%), Coagulase negative Staphylococcus (CONS) 32(1.3\%), respectively as shown in Table1.

Table 1: The frequency of microorganisms isolated from patients admitted in ICUS of COMS-TH,Bharatpur

\begin{tabular}{llcc}
\hline No. & Microorganisms & Total isolates & Percentage \\
\hline 1 & P.aeruginosa & 819 & $(35.4)$ \\
2 & K.pneumoniae & 637 & $(27.5)$ \\
3 & A. anitratus & 368 & $(15.9)$ \\
4 & Enterobacter Spp. & 144 & $(6.2)$ \\
5 & E.coli & 128 & $(5.5)$ \\
6 & P.mirabilis & 81 & $(3.5)$ \\
7 & Staphylococcus aureus & 53 & $(2.2)$ \\
8 & CONS & 32 & $(1.3)$ \\
9 & Candidaalbicans & 24 & $(1.0)$ \\
10 & Klebsiella oxytoca & 10 & $(0.4)$ \\
11 & Enterococcus spp & 08 & $(0.3)$ \\
12 & Citrobacter spp & 08 & $(0.3)$ \\
\multicolumn{2}{r}{ Total } & $\mathbf{2 , 3 1 2}$ & $\mathbf{( 1 0 0 \% )}$ \\
\hline
\end{tabular}


Journal of College of Medical Sciences-Nepal, 2012, Vol-8, No-3

The antibiotic sensitivity pattern of $P$. aeruginosa shows that almost all the isolates are resistant to Cephalexin (97.80\%). Among P.aeruginosa, tobramycin has the highest susceptibility rate (95.8\%) followed by meropenem (92.1\%) carbenecillin (82.9\%) and amikacin (74.8\%) and is depicted in Table 2.

Table 2 : Antibiotic pattern of P. aeruginosa:

\begin{tabular}{lcccc}
\hline Antibiotics & Sensitive (No.) & \% & Resistant (No.) & \% \\
\hline Cephalexin & 18 & 2.2 & 801 & 97.8 \\
Tobramycin & 785 & 95.8 & 34 & 4.2 \\
Piperacillin & 308 & 37.6 & 511 & 62.4 \\
Carbenecillin & 679 & 82.9 & 140 & 17.1 \\
Cefotaxime & 610 & 74.5 & 209 & 25.5 \\
Amikacin & 523 & 63.8 & 296 & 36.2 \\
Ceftriaxone & 401 & 48.9 & 418 & 51.1 \\
Ceftazidime & 488 & 59.5 & 331 & 40.5 \\
Gentamicin & 372 & 45.5 & 447 & 54.5 \\
Ciprofloxacin & 161 & 19.6 & 658 & 80.4 \\
Levofloxacin & 584 & 71.4 & 235 & 28.6 \\
Meropenem & 754 & 92.1 & 65 & 7.9 \\
\hline
\end{tabular}

Most of the frequently isolated organisms like K.pneumoniae, Acinetobacter anitratus,Enterobacter are highly resistant to ampicillin, cephalexin, cefepime, ciprofloxacin etc as shown in Table 3.

Table 3: Antibiotic resistance pattern of predominant microorganisms isolated from the specimens of the patients admitted in ICUs of CMS-TH (in \%)

\begin{tabular}{|c|c|c|c|c|c|c|c|}
\hline Antibiotics & $\begin{array}{c}\text { K.pneumoniae } \\
=637\end{array}$ & $\begin{array}{l}\text { A. anitratus } \\
=368\end{array}$ & $\begin{array}{l}\text { Enterobacter } \\
\text { spp. } \quad n=144\end{array}$ & $\begin{array}{l}\text { E.coli } \\
n=128\end{array}$ & $\begin{array}{c}\text { P.mirabilisn } \\
=81\end{array}$ & $\begin{array}{c}\text { S.aureus } \\
n=53\end{array}$ & $\begin{array}{l}\text { CONS } \\
\mathrm{n}=32 \\
\end{array}$ \\
\hline Ampicillin & 98.2 & 99.2 & 86.7 & 83.0 & 89.8 & 90.0 & 93.8 \\
\hline \multicolumn{8}{|l|}{ Amoxycillin/ } \\
\hline Clavulinic acid & 81.7 & 91.4 & 80.7 & 79.2 & 81.4 & 76.2 & 85.3 \\
\hline Ciprofloxacin & 69.4 & 78.2 & 67.2 & 66.2 & 71.6 & 81.1 & 89.7 \\
\hline Amikacin & 28.3 & 38.7 & 24.9 & 47.3 & 38.2 & 41.0 & 56.4 \\
\hline Gentamicin & 87.2 & 75.9 & 55.1 & 41.5 & 56.3 & 32.3 & 48.0 \\
\hline Cotrimoxozole & 51.8 & 44.8 & 34.0 & 26.8 & 68.1 & 38.4 & 57.3 \\
\hline Cefepime & 64.3 & 87.3 & 79.1 & 51.0 & 57.5 & 70.8 & 61.7 \\
\hline Ceftrixone & 78.5 & 51.8 & 59.3 & 52.4 & 68.7 & 38.5 & 32.3 \\
\hline Cephalixin & 95.7 & 95.5 & 96.8 & 76.3 & 71.2 & 98.0 & 96.2 \\
\hline Meropenem & 26.2 & 36.3 & 24.6 & 11.9 & 18.7 & 20.5 & 36.2 \\
\hline Levofloxacin & 38.2 & 27.4 & 37.3 & 21.8 & 48.1 & 24.3 & 38.0 \\
\hline Vancomycin & - & - & - & - & - & 0 & 0 \\
\hline Penicillin & - & - & - & - & - & 100 & 98.5 \\
\hline Cefotaxime & 68.4 & 71.8 & 61.6 & 31.7 & 60.9 & 58.3 & 55.9 \\
\hline
\end{tabular}


RK Sanjana et al. Microbial infection and antibiotic patterns among

\section{Discussion}

Infection among ICU patients might be Community acquired (bacterial meningitis / encephalitis pneumonia, endocarditis, intra-visceral abscesses urinary tract infection-UTI) or hospital and health care associated infection (surgical site infections, hospital acquired pneumoniae, catheter related blood stream infections, cather associated UTI). ${ }^{13}$ Modern intensive care is a multi-disciplinary effort of a team lead by the intensivist, clinical microbiologist and supported as and when required by associated medical/surgical specialities. A sustained parternership between the intensivist and the clinical microbiologist is essential for improving clinical outcome and optimising resource utilisation.

This article is an attempt to summarise the strategic patterns of the organisms and its resistance pattern that are isolated from the patients admitted in various ICUs in this tertiary care hospital.

Our study revealed that P.aeruginosa is the most common organism (35.4\%) followed by K. pneumonia (27.5\%) and A. anitratus (15.9\%). Many studies on the bacterial population in ICUs reported P.aeruginosa as the premier isolate. Study done by Deep Ganeja et al ${ }^{14}$ and also by Lokhart et a ${ }^{15}$ reported $\mathrm{P}$. aeruginosa as the most common isolate from lower respiratory tract specimen in ICU, but Kumari et al ${ }^{16}$ reported non fermenting gram negative bacilli other then P.aeruginosa as the most common pathogen. In another study by R.B Patwardhan el al ${ }^{17}$ reported Acinetobacter to be the most predominent isolate form ICUs acquired infection.

In this study high level of resistance was observed to cephalexin (97.8\%) ciprofloxacin (80.3\%), pipercillin (62.3\%) and gentamicin (54.5\%) against the most common isolate P.aeruginosa. Tobramycin, meropenem, carbenecillin and amikacin were found to be relatively effective against P.aerugiosa. High rate of resistance to cephalexin and quinolons among $P$. aeruginosa was in concord with the study done by Maksum Radji et al. ${ }^{8}$

In our studies, Pseudomonas, Klebsiella pneumoniae, Acinetobacter spps were multi drug resistant bacteria to ampicillin, cephalosporins including cefepime and quinolone antibiotics. These isolates are also resistant to gentamicin. Similar observations were demonstrated by sofianou DC et al ${ }^{18}$ and by Goel N. et a ${ }^{19}$ who revealed (96-100\%) resistance by P.aeruginosa and $\mathrm{K}$. pneumoniae to these drugs.This finding is related most probably due to extensive usage of cephalosporins and quinolones in this hospital and in other hospitals.

Another observation in this study is that amikacin, tobramycin \& meropenem showed good sensitivity against all bacteria isolated from ICU admitted patients. Navneeth et a $\mathrm{a}^{20}$ in 2002 and Jafari et al ${ }^{21}$ also reported good sensitivity to aminoglycosides against gram negative bacilli from the lower respiratory tract specimen of the ICU-admitted patients.

High sensitivity rate of meropenem which is similar with our finding is also reported by Deep Ganeja et el ${ }^{14}$ during a study in 2011 from the patients in ICUs. Shehabi et $\mathrm{al}^{22}$ and Lokhart et $\mathrm{al}^{15}$ also reported carbapenem as the most effective drug for patients in ICUs.

Similar studies were conducted in hospital and several ICUs in Asian countries including

Philippine $^{26}$, India ${ }^{19,}$ 27-30 and Nepal ${ }^{31}$ where P. aeruginosa, Acinetobacter spps., K. pneumoniae and 
Journal of College of Medical Sciences-Nepal, 2012, Vol-8, No-3

E. coli are the most frequent pathogens isolated. In all these studies, high resistance rate to cephalosporins \& quinolone antibiotics to these frequently isolated pathogens were reported.

Antibiotic use contributes to the emergence of antimicrobial resistance in gram positive as well as gram negative bacteria. ${ }^{23,24,25}$

\section{Conclusion}

P. aeruginosa, K .pneumoniae, Acinetobacter spps were the most frequently isolated pathogens in ICU patients in this hospital. Most of these frequent isolates are multidrug resistant organism. Majority of P. aeruginosa and also most of the isolates of $\mathrm{K}$. pneumonia and Acinetobacter spp are resistant to ampicillin, cephalosporins and quinolones as compared to aminoglycosides and meropenem in our study.

The prescribing of antibiotics in the ICUs is usually empiric. Therefore, the ongoing surveillance of antibiotic susceptibility patterns of predominant bacteria is a fundamental effort to monitor changes in susceptibility pattern and to guide the clinician in choosing empirical or directed therapy appropriately in the ICU setting.

Appropriate antibiotic utilization in ICU is crucial not only in ensuring an optimal outcome, but also in preventing the emergence of multi drug resistance bacteria.

\section{References}

1. Mullgan ME, Murray, Leisure K A, et al.Methicillin resistant staphylococcus aureus:A consensus review of the microbiology, pathogenesis and epidemiology with implication for prevention and management, $A m \mathrm{~J}$ Med 1993; 94:313-28.

2. Handwerger S, Raucher B, Altarac D, et al , Nosocomial outbreak due to Entrococcus faecium highly resistant to Vancomycin , penicillin and Gentamicin ,Clin Infect Dis 1993: 16: 750-5.

3. Chang VT, nelson K. The role of physical proximity in nosocomiocal diarrhoea. Clin Infect Disease 2000; 31:717-22.

4. Byers KE, Durbin LJ, Simonton BM et al, .Dissinfection of hospital rooms contaminated with Vancomycin resistant entrococcus faecium, Infect Control Hosp Epidemiol 1998: $19: 261-4$.

5. Myfleld JL, Leet T, Miller J, et al, .Environmental control to reduce transmission of clostridium difficile. Clin infect Dis 2000; 31:717-22.

6. Vincent JL , Rello J, Marshall J , et al . International study of the Prevalence and outcomes of infection in intensive care units JAMA 2009:302(21):2323-9.

7. Shulman L, Ost D Managing infection in the Critical care unit: How can infection control make the ICU safe, Crit Care Clin 2005:21:111-28.

8. Maksum Radji, Siti Fauziah, Nurgani Aribinuko. Antibiotic sensitivity pattern of bacterial pathogens in the intensive care unit of Fatmawati Hospital, Indonesia, Asian Pac J of Trop Biomedicine 2011;169(11):39-42.

9. Fridkin SK.Increasing Prevalence of Antimicrobial resistance in intensive care units .Crit Care Med 2001: 29: 64-8.

10. Jafri NJ, Ranjbar R, Haghi -Ashtiani MT, the Study of prevalence and antimicrobial susceptibility of tracheal bacterial stains isolated from pediatric pientients. Pak J bio Sci 2009:12:455-8. 
RK Sanjana et al. Microbial infection and antibiotic patterns among

11. Forbes BA, Sahm DF, Weissfeld AS, editors, Bacterial identification flow charts and schemes :A guidw to part III : In : Bailey and Scott’s diagnostic microbiology . 12 th /ed Missori: Mosby Elsevier; 2007;251-3.

12. Clinical and Laboratory Standard Institute. Permance standards for antimicrobial susceptibility: Sixteenth informational Supplement, Wayne, PA, USA: CLSI: 2006.M100-516.

13. Vincent JL,Bihri DJ ,Suter PM, et al, Nicolas -Chanoin $\mathrm{MH}$,et al .The prevalence of nosocomial infection in intensive care units in Europe .Results of the European Prevalence of Infection in Intensive care (EPIC ) Study. EPIC International Advisory committee, JAMA 1995;274:639-44.

14. Gagneja D, Goel N, Aggarwal R, et al changing trend of antimicrobial resistance among gram-negative bacilli isolated from lower respiratory tract of ICU patients: A 5 years study. Indina J Crit Care Mad 2011;15:164-7.

15. Lockhart SR,Abramson MA,Beekmann SE,et al Antimicrobial resistance amoung gram-negative bacilli causing infections in intensive care unit Patients in the United States between 1993 and 2004, J Clin Microbiol 2007; 45:3352-9.

16. Kumari HB, Nagarathna S,Chandramuki A. Antimicrobial resistance pattern amoung gram negative bacilli of lower respiratory tract specimens of intensive Care unit patients in a neurocentre, Indian J Chest Dis Allied Sci 2007;49:19-22.

17. A study on nosocomial pathogens in icu with special reference to multirsistant AcinetobacterBaumannii harbouring multipleRBPatwardhan, P.K. Dhakephalkart, K,B Niph DKar And B A ChopadeIndian J Med Res 128, Auguest. 2008;178-87.
18. Sofianou DC, Constandinidis TC, Yannacou M. Anastasiou H, Sofinas E, Analysis of risk factors for ventilator associated pheumonia in a multidisciplinary intensive care unit Eur J Clin Microbial infect Dis 2000;19:460-3.

19. Goel N Chaudhary U.Aggarwal R,Bala K.Antibiotic sensitivity pattern of gram negative bacilli isolated from the lower respiratory tract of ventilated patients in the intensive care units Indan J Crit Care Med 2009;13:148-51.

20. Navaneeth BV, Belwadi MR, Antibiotic resistance amoung gram graDIs Allied Sci 2002;44:173-6.

21. Jafari NJ ,Ranjbar R, Highi -Ashtiani MT,Abedini M,Izadi M The study of prevalence and antimicrobial susceptility of tracheal bacterial stains isolated from pediatric patients pak J bio Sci 2009;12:455-8.

22. Shehabi AA,Baadran I Microbial Infection and antibiotic resistance patterns amoung Jordanian intensive care patients, East Mediterr Health J. 1996:2:515-20.

23. Bronzwaer SL,Cars O,Buchholz U,Molsted SGoettsch W,Veldhuijzen IK,Et al .A Eurpean study on the relationship between antimicrobial use and antimicrobial resistance Emerg Infect Dis 002;8:278-82.

24. Beekmann SE,Heilmann KP,Richter SS.Antimicrobial resistance in streptococcus pheumoniae,Haemophilus influnzae ,Marxella catarrhals and group A beta Heamolytic streptococci in 2002-2003 int J Antimicrobial a Agents2005: 25:148-56.

25. Erb A Sturner $\mathrm{T}$ Brenner H Prevalence of Antibiotic resistance in Escherichia : overview of geographical, temporal and methodological variations Eur j Clin Microbial infect Dis 2007;26:83-90. 
Journal of College of Medical Sciences-Nepal, 2012, Vol-8, No-3

26. Elizow JM Gil CJ Mantaring JB ,Fox MP, Maclood WB,Mendoza M ,et al High frequency of Multidrug resistance gram negative rods in 2 neonatal intensive care units in the Philippines Infect Control Hosp Epidemiology 2008;30:(6): 543-9.

27. Shalni S Kranthi K,Gopalkrishna Bhat K Microbiological profile of Nosocomiocal infection in the intensive care unit J Clin Diagnostic Res 2010;4(5);3109-012.

28. Kaul S.Brahmadathan KN, Jagannati M, Sudaraam TD, et al. One year trends in the gram negative bacterial antibiotic susceptibility patterns in a Medical intensive care unit in south India Indian $J$ Med Microbial 2007;25:230-5.
29. Tsering DC, Das S Adhiakri I Pal R Singh TS Extended spectrum beta-lactamase detection in gram -negative bacilli of nosocomiocal Origen J Globinfect Dis 2009; 1:87-92.

30. Kumari HB,Nagarathna S.Chandrumuki A. Antimicrobial restantance pattern Among aerobic gram negative bacilli of lower respiratory tract Specimens of intensive care unit patients in a neurocentre. Indian J Chist Dis Allied Sci 2007:49:19-22.

31. Shankar PR, Partha P, Dubey AK, Intensive care unit drug utilization in a teaching hospital in Nepal. Kathmandu Univ Med J 2005: 3(2): 130-7. 